\title{
Long-term follow-up of 46 cases of primary fallopian tube carcinoma: a single institute study
}

\author{
Shanji Li ${ }^{1,2 \#}$, Minhua Yu ${ }^{1,2 \#}$, Wenxin Bai ${ }^{1,3}$, Jun Shi ${ }^{1,2}$, Wen $\mathrm{Di}^{1,2,4}$ \\ ${ }^{1}$ Department of Obstetrics and Gynecology, Ren Ji Hospital, School of Medicine, Shanghai Jiao Tong University, Shanghai, China; ${ }^{2}$ Shanghai Key \\ Laboratory of Gynecologic Oncology, Ren Ji Hospital, School of Medicine, Shanghai Jiao Tong University, Shanghai, China; ${ }^{3}$ School of Medicine, \\ Shanghai Jiao Tong University, Shanghai, China; ${ }^{4}$ State Key Laboratory of Oncogenes and Related Genes, Shanghai Cancer Institute, Ren Ji \\ Hospital, School of Medicine, Shanghai Jiao Tong University, Shanghai, China \\ Contributions: (I) Conception and design: S Li, W Di; (II) Administrative support: M Yu; (III) Provision of study materials or patients: W Bai; (IV) \\ Collection and assembly of data: S Li, J Shi; (V) Data analysis and interpretation: S Li, W Di; (VI) Manuscript writing: All authors; (VII) Final \\ approval of manuscript: All authors. \\ \#These authors contributed equally to this work. \\ Correspondence to: Wen Di, MD, PhD. Department of Obstetrics and Gynecology, Ren Ji Hospital, School of Medicine, Shanghai Jiao Tong \\ University, Shanghai, China. Email: diwen163@163.com.
}

Background Primary fallopian tube carcinoma (PFTC) is a rare malignancy. In recent years the incidence of PFTC has been rising. This study retrospectively analyzed 46 cases of PFTC to identify prognostic factors that may impact the survival of patients with PFTC and explored the clinical characteristics.

Methods: The clinical data of patients who had undergone surgery and adjuvant chemotherapy in Ren Ji Hospital, School of Medicine, Shanghai Jiao Tong University from 1995 to 2015 were retrospectively analyzed. We analyzed clinical data. Cox proportional hazards model was used for univariate and multivariate survival analysis.

Results: The level of CA125 increased in almost all patients with advanced-stage (stage III-IV) carcinoma and about half the patients with early stage (stage I-II) carcinoma. On ultrasound examination, 41 cases had pelvic mass, and five cases had intrauterine space-occupying lesion. Nine patients underwent curettage (19.6\%). By the International Federation of Gynecology and Obstetricians (FIGO) staging system, the distribution of patients was 18 at stage I, 16 at stage II, 10 at stage III, and 2 at stage IV. The mainstay of treatment was surgical cytoreduction and platinum-based chemotherapy. Four patients had residual tumors diameter $\leq 1 \mathrm{~cm}(\mathrm{R} 1), 10$ had residual tumors diameter $>1 \mathrm{~cm}$, and 32 had no macroscopic residual tumor (R0). Forty patients received postoperative intravenous (IV) chemotherapy. The five-year overall survival (OS) was $94.7 \%$ in stage I, $80.0 \%$ in stage II, $44.4 \%$ in stage III, and $0 \%$ in stage IV. Univariate and multivariate analysis revealed that residual tumor was independent prognostic variable for OS. Univariate and multivariate analysis revealed that ascites tumor cells and residual tumor were independent prognostic variables for progression free survival (PFS).

Conclusions: Any postmenopausal women with vaginal bleeding, vaginal discharge, or lower abdominal pain should be alert to PFTC. Complete tumor markers and imaging examination should be conducted as soon as possible to improve the early diagnosis rate of the disease. Regardless of whether the operation is a comprehensive staging operation or cytoreductive surgery (CRS), achieving satisfactory R0 can improve OS and PFS. It is important the ascitic fluid is tested for tumor markers in order to predict PFS.

Keywords: Primary fallopian tube carcinoma (PFTC); prognosis; follow-up; overall survival; progression free survival

Submitted Jun 02, 2021. Accepted for publication Aug 12, 2021.

doi: 10.21037/apm-21-2083

View this article at: https://dx.doi.org/10.21037/apm-21-2083 


\section{Introduction}

Primary fallopian tube carcinoma (PFTC) is a rare malignant tumor of the female reproductive system and accounts for $0.14 \%$ to $1.8 \%$ of genital tract malignancy $(1,2)$. Renaud first described it in an unpublished manuscript in the library of The Royal College of Surgeons in 1847. Until 1866, Orthomann carried out the complete description of PFTC, attracting attention $(3,4)$. Epidemiological research showed a different incidence of PFTC in different countries from 1.2/1,000,000 to $6.7 / 1,000,000$ per year (5-10). Recent studies elucidated that most high-grade serous ovarian cancer originates from the fallopian tube and serous tubal intraepithelial carcinoma (STIC) spreads to the ovaries or peritoneum $(7,9)$. However, STIC incidence and early-stage PFTC have not been comprehensively assessed until now (11). In addition, the clinical and morphological features of late stage PFTC are so similar to epithelial ovarian cancer (EOC) and primary peritoneal carcinoma that it is difficult to distinguish even at the time of operation (8). Thus, the true incidence of PFTC was probably underestimated in previous years. Due to the rarity of this disease, the literature on PFTC is limited. We conducted a retrospective study of 46 cases of PFTC to identify the prognostic factors and to explore the clinical characteristics of this disease. We present the following article in accordance with the STROBE reporting checklist (available at https://dx.doi.org/10.21037/apm-21-2083).

\section{Methods}

We enrolled 46 patients who had confirmed PFTC from 1995 to 2015 at Ren Ji Hospital, School of Medicine, Shanghai Jiao Tong University. All patients underwent surgeries followed by chemotherapies without radiotherapy. All procedures performed in this study involving human participants were in accordance with the Declaration of Helsinki (as revised in 2013). The study was approved by ethic committee of Ren Ji Hospital, School of Medicine, Shanghai Jiao Tong University (NO.20200704) and informed consent was taken from all the patients.

Clinical history collected included age, menopausal status, time of pregnancy and childbirth, clinical symptoms, preoperative serum carbohydrate antigen 125 (CA125) level, preoperative imaging data, ascitic fluid specimen, clinicopathological staging, cytoreductive surgery (CRS), chemotherapy, and follow-up.
All patients were staged according to the International Congress of Gynecology and Obstetricians (FIGO) staging (12). Histological evaluation is based on the World Health Organization (WHO) classification of malignant epithelial fallopian tube carcinoma (13). Recurrence is defined as increased CA125 and/or imaging findings of the relapsed tumor. Progression-free survival time (PFS) refers to the initial treatment to the progression of the tumor or progression to the final follow-up time. Overall survival (OS) is the time from the start of treatment to patient death from the tumor or to the last follow-up time.

\section{Statistical analysis}

SPSS 26.0 software was used for statistical analysis. Singlefactor analysis of survival curves was performed using the Kaplan-Meier method. The Log-rank test was used to determine the significance of survival distribution in each group. Multivariate analysis was calculated using the Cox proportional hazards model. $\mathrm{P}<0.05$ was defined as statistically significant.

\section{Results}

\section{Clinical characteristics (shown in Table 1)}

\section{Basic information}

The mean age of the 46 patients was $60.6 \pm 10.8$ years (range, 39 to 82 years) at the time of symptom onset. The median age was 60.6 years. There were 36 cases $(78.3 \%)$ who were postmenopausal. In 43 cases (93.5\%), the average number of pregnancies was 3.1, and the average number of births was 1.9. Three patients $(6.5 \%)$ had not given birth. Two patients had undergone prior tubal ligation, one patient who had undergone unilateral tubal oophorectomy due to ovarian cyst, one patient who had accepted hysterectomy due to hysteromyoma, and one patient who had a history of breast cancer surgery.

\section{Clinical manifestation}

Clinical symptoms included vaginal bleeding (30 cases, $65.2 \%$ ), vaginal discharge (nine cases, $19.6 \%$ ), abdominal pain (eight cases, $17.4 \%$ ), abdominal distension (five cases, $10.9 \%$ ), urinary urgency (two cases, $4.3 \%$ ), waist soreness (two cases, $4.3 \%$ ), and palpable mass (one case, $2.2 \%$ ). The typical Latzko triad (vaginal bleeding/discharge, abdominal pain, and pelvic mass) was rare (one case, $2.2 \%$ ). 
Table 1 Clinical and pathological characteristics of 46 patients diagnosed with PFTC

\begin{tabular}{|c|c|c|}
\hline Characteristics & No. & $\begin{array}{c}\text { Percentage } \\
(\%)\end{array}$ \\
\hline Age/year (average, range) & 60 [39-82] & \\
\hline \multicolumn{3}{|l|}{ Menopause } \\
\hline Yes & 36 & 78.3 \\
\hline No & 10 & 21.7 \\
\hline \multicolumn{3}{|l|}{ Nulliparous } \\
\hline Yes & 3 & 6.5 \\
\hline No & 43 & 93.5 \\
\hline \multicolumn{3}{|l|}{ Tubal ligation history } \\
\hline Yes & 2 & 4.3 \\
\hline No & 44 & 95.7 \\
\hline \multicolumn{3}{|l|}{ Symptom } \\
\hline Vaginal bleeding & 30 & 65.2 \\
\hline Vaginal discharge & 9 & 19.6 \\
\hline Abdominal pain & 8 & 17.4 \\
\hline Abdominal distension & 5 & 10.9 \\
\hline Urinary urgency & 2 & 4.3 \\
\hline Waist soreness & 2 & 4.3 \\
\hline Palpable mass & 1 & 2.2 \\
\hline Combination & 16 & 34.8 \\
\hline Latzko triad symptom & 1 & 2.2 \\
\hline None & 4 & 8.7 \\
\hline \multicolumn{3}{|c|}{ Preoperative serum CA125 (U/mL) } \\
\hline$<35$ & 15 & 32.6 \\
\hline$\geq 35$ & 27 & 58.7 \\
\hline Unknown & 4 & 8.7 \\
\hline \multicolumn{3}{|l|}{ Imaging findings } \\
\hline Abdominal or pelvic mass & 41 & 89.1 \\
\hline Endometrial neoplasms & 5 & 10.9 \\
\hline \multicolumn{3}{|l|}{ Diagnostic curettage } \\
\hline Yes & 9 & 19.6 \\
\hline No & 37 & 80.4 \\
\hline \multicolumn{3}{|l|}{ Histologic subtype } \\
\hline High grade serous & 39 & 84.8 \\
\hline Endometrial & 2 & 4.3 \\
\hline Transitional cell & 1 & 2.2 \\
\hline
\end{tabular}

Table 1 (continued)
Table 1 (continued)

\begin{tabular}{|c|c|c|}
\hline Characteristics & No. & $\begin{array}{c}\text { Percentage } \\
(\%)\end{array}$ \\
\hline Neuroendocrine carcinoma & 1 & 2.2 \\
\hline Immature teratoma & 1 & 2.2 \\
\hline Choriocarcinoma & 2 & 4.3 \\
\hline \multicolumn{3}{|l|}{ FIGO stage } \\
\hline I & 18 & 39.1 \\
\hline II & 16 & 34.8 \\
\hline III & 10 & 21.7 \\
\hline IV & 2 & 4.3 \\
\hline \multicolumn{3}{|l|}{ Ascites tumor cells } \\
\hline Positive & 11 & 23.9 \\
\hline Negative & 22 & 47.8 \\
\hline Unknown & 13 & 28.3 \\
\hline \multicolumn{3}{|l|}{ Surgical method } \\
\hline $\begin{array}{l}\text { Comprehensive staging } \\
\text { operation }\end{array}$ & 19 & 41.3 \\
\hline Debulking surgery & 25 & 54.3 \\
\hline Salpingectomy & 2 & 4.3 \\
\hline \multicolumn{3}{|l|}{ Residual disease, $\mathrm{cm}$} \\
\hline$\leq 1$ & 36 & 78.3 \\
\hline$>1$ & 10 & 21.7 \\
\hline \multicolumn{3}{|l|}{ Lymphadenectomy } \\
\hline Yes & 25 & 54.3 \\
\hline No & 21 & 45.7 \\
\hline \multicolumn{3}{|l|}{ Omentectomy } \\
\hline Yes & 33 & 71.7 \\
\hline No & 13 & 28.3 \\
\hline \multicolumn{3}{|l|}{ Appendectomy } \\
\hline Yes & 23 & 50.0 \\
\hline No & 23 & 50.0 \\
\hline \multicolumn{3}{|l|}{ Primary IP chemotherapy } \\
\hline Yes & 22 & 47.8 \\
\hline No & 24 & 52.2 \\
\hline \multicolumn{3}{|l|}{ TP chemotherapy } \\
\hline$\geq 6$ courses & 24 & 52.2 \\
\hline$<6$ courses & 22 & 47.8 \\
\hline
\end{tabular}




\section{Laboratory examination}

The serum CA125 was detected in 42 cases pre-operation (91.3\%), of which 27 cases were elevated ( $\geq 35 \mathrm{U} / \mathrm{L}, 58.7 \%$ ), 15 cases were normal level (32.6\%), and four cases had not been detected or unknown (8.7\%).

\section{Imaging examination}

All patients underwent gynecological pelvic ultrasound examination, in which 41 cases $(89.1 \%)$ had pelvic masses, and five cases $(10.9 \%)$ where the pelvic mass was found in the uterine cavity. Most of the pelvic masses were solid or cystic, and blood flow signals were detected in the solid part of the pelvic mass. Due to intrauterine space occupation or postmenopausal bleeding, nine patients underwent curettage before surgery (19.6\%).

\section{Treatment}

\section{Surgical treatment}

The initial treatment for all 46 patients was surgery. One patient received two circles of arterial intubation chemotherapy before the operation. Among the 33 patients who underwent ascites cytology examination, cancer cells were found in 11 cases (33.3\%). Hysterectomy with bilateral salpingo-oophorectomy was undergone in 42 patients, and one patient who received bilateral salpingo-oophorectomy only. Due to the advanced stage of the tumor, one patient underwent palliative surgery of unilateral salpingooophorectomy, and two patients received unilateral salpingooophorectomy due to choriocarcinoma. Omentectomy was carried out in 33 cases, among which pathology revealed metastasis in four cases. Except that 1 patient had received appendectomy before, appendectomy was carried out in 23 cases, and one patient had undergone a prior appendectomy. No metastasis was found in the postoperative pathology. Pelvic lymphadenectomy was undertaken in 25 cases, in two of which metastasis was found. Paraaortic lymphadenectomy was performed in eight patients, with metastasis was found in three cases. A comprehensive staging operation was performed in 19 cases, and debulking surgery was performed in 25 cases. As for postoperative residual tumor size, no macroscopic residual tumor (R0) was found in 32 cases, four cases had residual tumor diameter $\leq 1 \mathrm{~cm}$ (R1), and 10 cases had residual tumor diameter $>1 \mathrm{~cm}$.

\section{Histologic subtype}

All histologic subtypes of the 46 patients were confirmed by pathology in our hospital, including 40 cases of high- grade serous carcinoma (87.0\%), one case of endometrial carcinoma $(2.2 \%)$, one case of neuroendocrine carcinoma $(2.2 \%)$, one case of transitional cell carcinoma $(2.2 \%)$, one case of immature teratoma $(2.2 \%)$, and two cases of choriocarcinoma $(4.3 \%)$. Serous adenocarcinoma of the fallopian tube combined with endometrioid adenocarcinoma was found in one case. There was also one case confirmed with serous adenocarcinoma of the fallopian tube combined with endometrial adenocarcinoma, as well as cervical squamous cell carcinoma.

\section{FIGO staging}

According to the FIGO staging classification for cancer of the ovary, fallopian tube, and peritoneum, there were 19 cases $(41.3 \%)$ in stage I, 15 cases $(32.6 \%)$ in stage II, 10 cases $(21.7 \%)$ in stage III, and two cases $(4.3 \%)$ in stage IV.

\section{Postoperative chemotherapy}

Intraperitoneal chemotherapy was performed in 22 cases and intravenous (IV) chemotherapy in 40 cases. IV chemotherapy was received more than six times in 24 patients, and 16 patients received IV chemotherapy less than six times. The three patients with FIGO stage I cancer did not receive chemotherapy due to advanced age, one patient with FIGO stage II cancer did not receive chemotherapy due to abnormal liver function, and two patients with FIGO stage II cancer refused chemotherapy.

IV TP (paclitaxel + cisplatin) or TC (paclitaxel + carboplatin) regimen was used after 2003, while IV PAC (cisplatin + adriamycin + cyclophosphamide) regimen was used before 2003. Bleomycin + etoposide (VP-16) + cyclophosphamide regimen was used for immature teratoma, penta-fluorouracil $(5-\mathrm{Fu})+$ dactinomycin $(\mathrm{KSM})$ regimen was used for choriocarcinoma, and VP-16 + cisplatin regimen was used for neuroendocrine carcinoma.

\section{Follow-up}

The average period of follow-up was 102.7 months until October 8, 2020. Two cases were lost to follow-up, and the follow-up rate was $95.7 \%$.

\section{Survival analysis}

At the end of the follow-up, 17 patients died, and the mortality rate was $37 \%$. The five-year OS rate was $73.9 \%$, and the five-year PFS rate was $67.4 \%$. The five-year OS rate was $94.7 \%$ in stage I, $80.0 \%$ in stage II, $44.4 \%$ in stage 
Table 2 Follow-up of mortality rate and five-year survival rate

\begin{tabular}{|c|c|c|c|}
\hline Stage & Number (proportion) & Death toll (mortality rate) & $\begin{array}{c}\text { Five-year survival number } \\
\text { (five-year survival rate) }\end{array}$ \\
\hline 1 & $19(41.3 \%)$ & $3(15.8 \%)$ & $18(94.7 \%)$ \\
\hline II & $15(32.6 \%)$ & $5(33.3 \%)$ & $12(80.0 \%)$ \\
\hline III & $9(21.7 \%)$ & $6(66.6 \%)$ & $4(44.4 \%)$ \\
\hline
\end{tabular}

Table 3 Follow-up of special pathological types

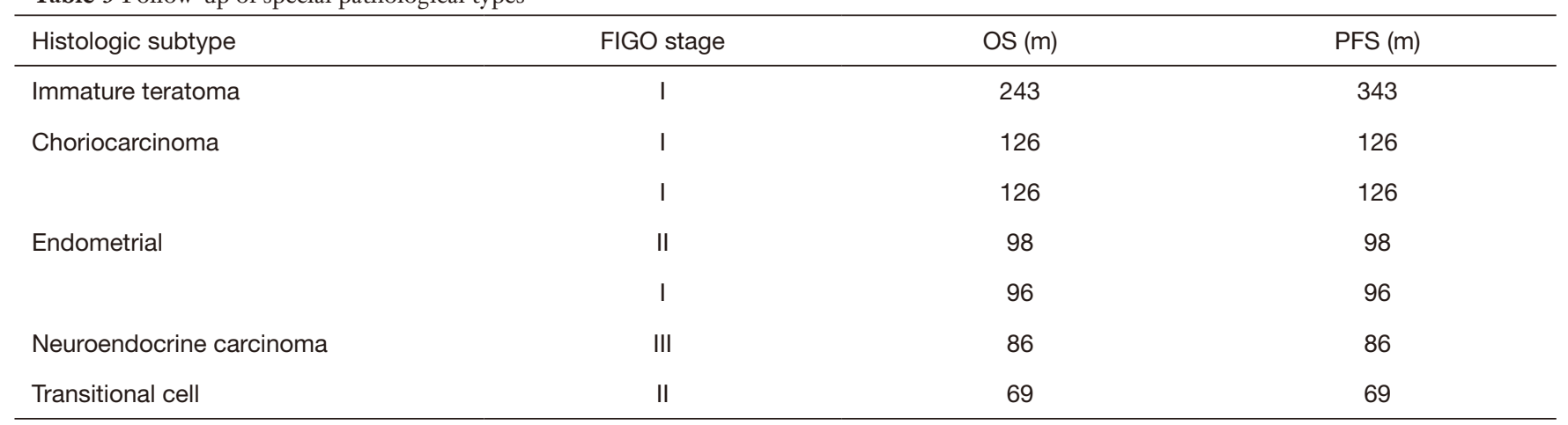

FIGO, International Congress of Gynecology and Obstetricians; OS, overall survival; PFS, progression-free survival.

III, and $0 \%$ in stage IV, as shown in Tables 2 and 3.

Univariate analysis showed that the prognostic factors affecting OS were age ( $\geq 60$ vs. $<60$ years, $\mathrm{P}=0.013)$, the postmenopausal period ( $\geq 10$ vs. $<10$ years, $\mathrm{P}=0.039)$, FIGO staging (III-IV vs. I-II, $\mathrm{P}=0.000$ ), surgical method (staged surgery vs. body surgery, $\mathrm{P}=0.029)$ and residual tumor ( $\geq 1$ vs. $<1 \mathrm{~cm}, \mathrm{P}=0.000$ ) (Table 4). The Cox proportional hazards model showed that residual tumor $(\mathrm{P}=0.002)$ was an independent prognostic factor of OS (Table 5).

Univariate analysis showed that the prognostic factors affecting PFS were age ( $\geq 60 v s$. $<60$ years, $\mathrm{P}=0.006)$, and the postmenopausal period ( $\geq 10$ vs. $<10$ years, $\mathrm{P}=0.012$ ), FIGO staging (III-IV vs. I-II, $\mathrm{P}=0.000$ ), ascites tumor cells (positive vs. negative, $\mathrm{P}=0.047$ ), surgical method (full staging and CRS, $\mathrm{P}=0.037)$ and residual tumor $(\geq 1$ vs. $<1 \mathrm{~cm}, \mathrm{P}=0.000)$ (Table 6). The Cox proportional hazards model showed that ascites tumor cells $(\mathrm{P}=0.030)$ and residual tumors $(\mathrm{P}=0.011)$ were independent prognostic factors for PFS (Table 5).

The OS and PFS curves of this queue are shown in Figure 1.

All the other factors like pretreatment of CA125 levels, histological type, and chemotherapy had no significant effect on the prognosis.

\section{Discussion}

PFTC is a rare malignant tumor of the female reproductive system. In recent years, with the continuous in-depth research on the origin of malignant pelvic tumors, more and more reports of fallopian tube carcinoma have been reported (14). PFTC were usually treated in various ways $(10,15,16)$. A single-center study of Fudan University Shanghai University Obstetrics and Gynecology Hospital collected 101 cases, and the longest follow-up time was 143 months (17).

The literature reports that the most common age for PFTC is 55-65 years old, mostly in postmenopausal women (1,10,15-17). An 88-year-old woman suffering PFTC is the oldest patient reported on to date (18). The cohort reported in our study included 46 patients with PFTC who had a mean age (60.6 years), similar to those in other studies, with most $(78.3 \%)$ of the patients being postmenopausal and having a mean spread of 16.1 years.

Ultrasound is an important examination tool. The sign for PFTC is that there is no echo or low echo in the adnexal area with papillary process or intraluminal wall mass present. However, the echo of most fallopian tube carcinomas is nonspecific, which is similar to other 
Table 4 Impact of prognostic factors on OS by univariate analysis in PFTC of 46 patients

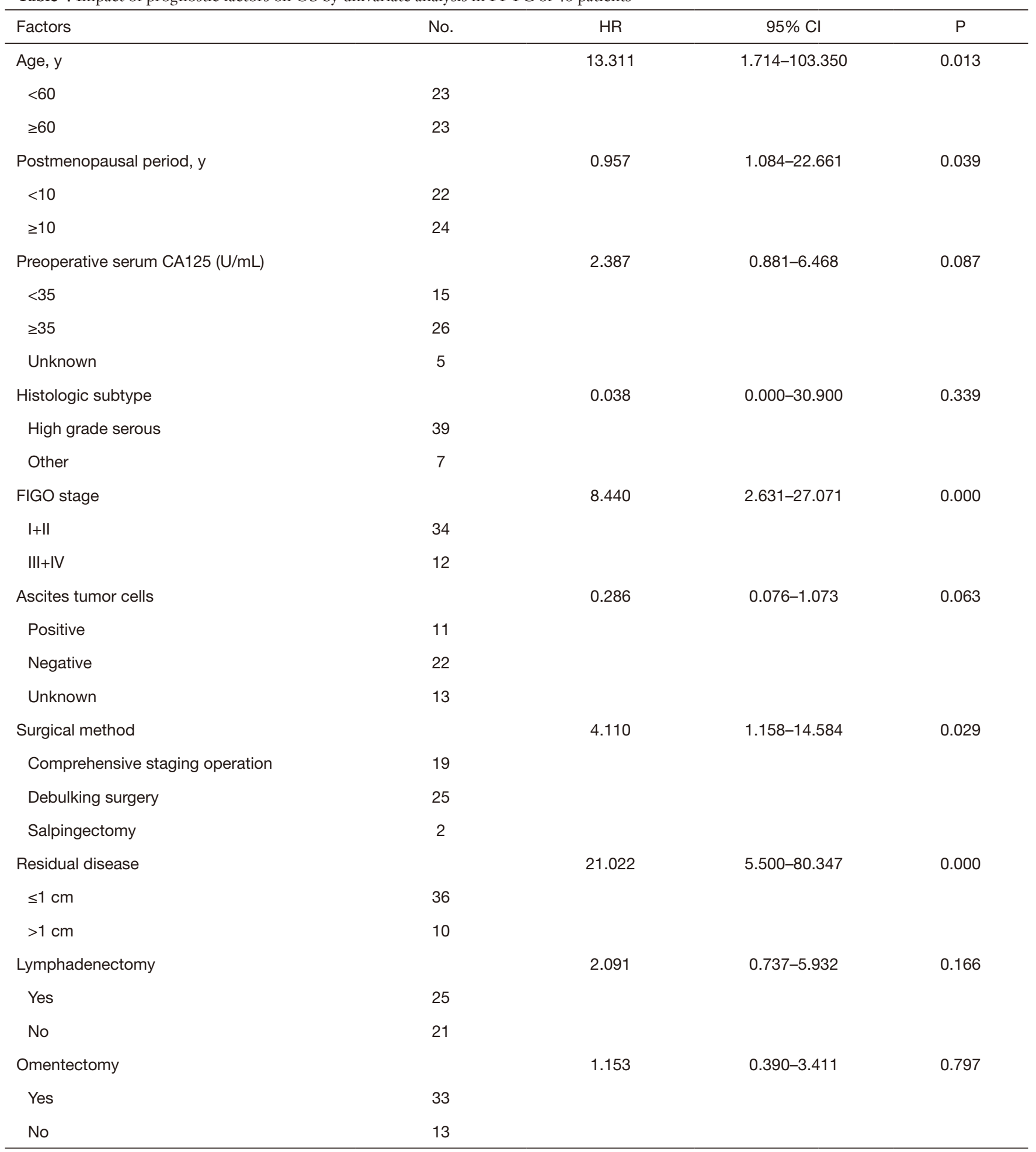

Table 4 (continued) 
Table 4 (continued)

\begin{tabular}{lccc}
\hline Factors & No. & HR & $95 \% \mathrm{Cl}$ \\
\hline Appendectomy & & 1.565 & $0.549-4.465$ \\
Yes & 23 & & 0.402 \\
No & 23 & & $0.237-1.972$ \\
Primary IP chemotherapy & & 0.683 & 0.481 \\
Yes & 22 & & $0.524-4.007$ \\
No & 24 & & 0.474 \\
IV chemotherapy & & & \\
$\geq 6$ courses & 24 & & \\
$<6$ courses & 22 & & \\
\hline
\end{tabular}

OS, overall survival; PFTC, primary fallopian tube carcinoma; FIGO, International Congress of Gynecology and Obstetricians; IV, intravenous; IP, intraperitoneal.

Table 5 Variables predictive of survival by Cox proportional hazards model in PFTC

\begin{tabular}{lccc}
\hline Variables & Wald $\chi^{2}$ & Risk ratio & $95 \% \mathrm{Cl}$ \\
\hline OS & & & $\mathrm{P}$ \\
FIGO stages & 1.700 & 2.290 & $0.659-7.955$ \\
Surgical method & 1.313 & 0.367 & $0.066-2.038$ \\
Residual disease, cm & 9.882 & 9.335 & $2.319-37.578$ \\
Postmenopausal period, y & 0.015 & 0.872 & $0.097-7.816$ \\
Age, y & 3.264 & 7.264 & 0.002 \\
PFS & & $0.845-62.434$ \\
FIGO stages & 0.578 & 2.011 & 0.071 \\
Ascites tumor cells & 4.708 & 0.049 & $0.332-12.184$ \\
Surgical method & 0.310 & $0.003-0.747$ \\
Residual disease, cm & 6.515 & 2.273 & $0.127-40.838$ \\
Postmenopausal period, y & 0.197 & 66.358 & 0.030 \\
Age, y & 0.002 & 0.526 & 0.077 \\
\hline
\end{tabular}

PFTC, primary fallopian tube carcinoma; OS, overall survival; FIGO, International Congress of Gynecology and Obstetricians; PFS, progression-free survival.

pelvic diseases such as ovarian cancer. PFTC differs from ovarian cancer in that the intrauterine mass is often seen by preoperative ultrasound, and diagnostic curettage is performed. In this study, all patients received gynecological pelvic ultrasound examinations. Of these, 41 cases $(89.1 \%)$ had a pelvic mass, five cases $(10.9 \%)$ had intrauterine spaceoccupying lesion.

CT and MRI examination can also help diagnosis, but the specificity is not high. When CT or MRI examination finds dachshund-like pelvic mass, intrauterine effusion, or hydrosalpinx, we should be alert to the possibility of fallopian tube cancer.

CA125 is still a common tumor index used in the monitoring of PFTC. In this study, elevated preoperative CA125 level was found in $57.5-85.7 \%$ of the patients and 27 patients (58.7\%) had preoperative CA125 level greater than 
Table 6 Impact of prognostic factors on PFS by univariate analysis in PFTC of 46 patients

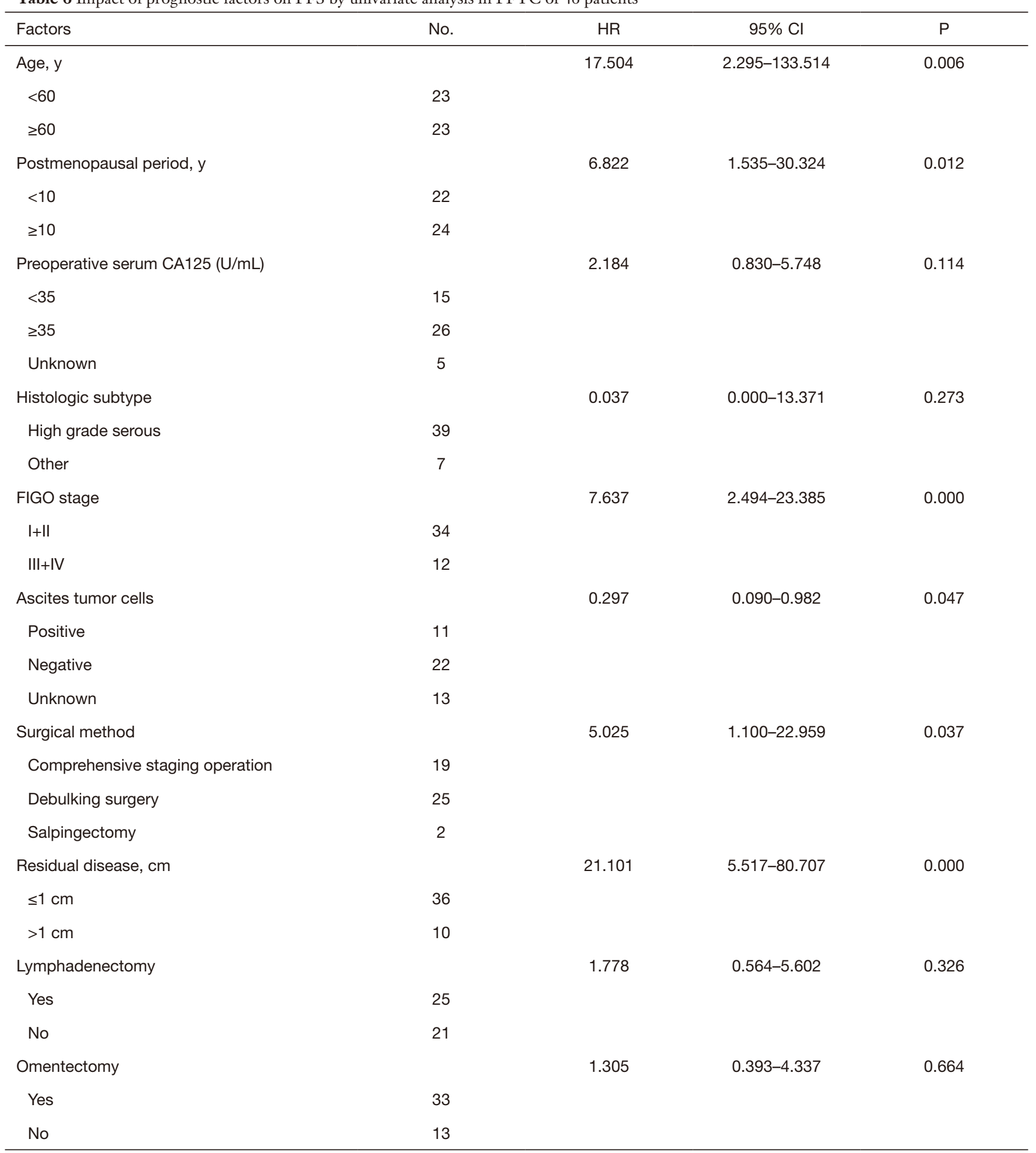

Table 6 (continued) 
Table 6 (continued)

\begin{tabular}{lccc}
\hline Factors & No. & HR & $95 \%$ Cl \\
\hline Appendectomy & & 2.349 & $0.707-7.801$ \\
Yes & 23 & & \\
No & 23 & & 0.163 \\
Primary IP chemotherapy & & 0.591 & $0.187-1.862$ \\
Yes & 22 & & 0.369 \\
No & 24 & & $0.584-5.808$ \\
IV chemotherapy & & 1.841 & 0.298 \\
$\geq 6$ courses & 24 & & \\
$<6$ courses & 22 & & \\
\hline
\end{tabular}

PFS, progression-free survival; PFTC, primary fallopian tube carcinoma; FIGO, International Congress of Gynecology and Obstetricians; IV, intravenous; IP, intraperitoneal.

$35 \mathrm{U} / \mathrm{mL}$. In the statistical analysis of tumor marker serum CA125, we found that there was a significant difference in preoperative serum CA125 levels between early (stage I-II) and late (stage III-IV) patients $(\mathrm{P}<0.001)$. In the early stage (stage I-II), there were 16 cases with elevated serum CA125, accounting for $53.3 \%(16 / 30)$; in the late stage (stage III-IV), there were 11 cases with elevated serum CA125, accounting for $91.7 \%$ (11/12), and four cases were unknown. The level of CA125 increased in almost all patients with advanced-stage (stage III-IV) and about half of the patients with early stage (stage I-II).

It isn't easy to diagnose PFTC before surgery. Preoperative transvaginal color Doppler ultrasound, CT, MRI, and diagnostic curettage pathology combined with detection of serum tumor markers are helpful for early diagnosis (19). In 2007, prophylactic salpingooophorectomies in BRCA+ women identified the fimbria as a site of origin for early serous carcinoma (STIC) (20). The STIC lesions either invade the tubal stroma locally (developing into PFTC) or exfoliate onto the surface of the ovary or peritoneum (primary ovarian or peritoneal carcinoma) (21). Although STIC lesions have been found with late-stage HGSC (high grade serous carcinoma) in up to $60 \%$ of cases, not all STIC lesions appear to progress to clinical HGSCs, nor would all HGSCs arise from STIC lesions, even in high-risk women (22). Therefore, the origin of pelvic serous carcinoma still needs a large sample prospective study (23).

Based on the FIGO classification system, endometrial carcinoma is divided into 1,2 , and 3 grades. Mucinous carcinoma is not classified. Clear cell carcinoma pain and undifferentiated carcinoma are considered high grade carcinoma. The pathological types of 46 patients in this study included 39 cases (84.8\%) of highgrade serous carcinoma similar to those previously described (6), two cases of endometrioid carcinoma, one case of transitional cell carcinoma, one case of neuroendocrine carcinoma, one case of immature teratoma, and two cases of choriocarcinoma. Although it has been reported that pathological grade is a very important prognostic factor for PFTC, in this study, serous carcinoma is considered high grade, so pathological grade is not discussed.

PFTC staging is based on surgical and pathological findings. In 1991, a system for staging PFTC was approved by the FIGO (24). Based on the progress of research in tumor biology and changes in clinical practice, ovarian cancer, fallopian tube cancer, and peritoneal cancer were classified into one category according to FIGO's surgical pathological staging system in 2013. The National Comprehensive Cancer Network (NCCN) guidelines, 2020, for PFTC treatment is also based on EOC.

In this study, 18 cases (39.1\%) were FIGO stage I, 16 cases $(34.8 \%)$ were FIGO stage II, 10 cases $(21.7 \%)$ were FIGO stage III, and two cases $(4.3 \%)$ were FIGO stage IV. The FIGO distribution of stages I and II was relatively similar to most studies in the literature (6). PFTC was more likely to be diagnosed with early-stage tumors and have an improved overall and cancer-specific survival rate compared with women with primary ovarian cancer (25). This may be due to lower abdominal pain resulting from tubal dilatation 
Data 1

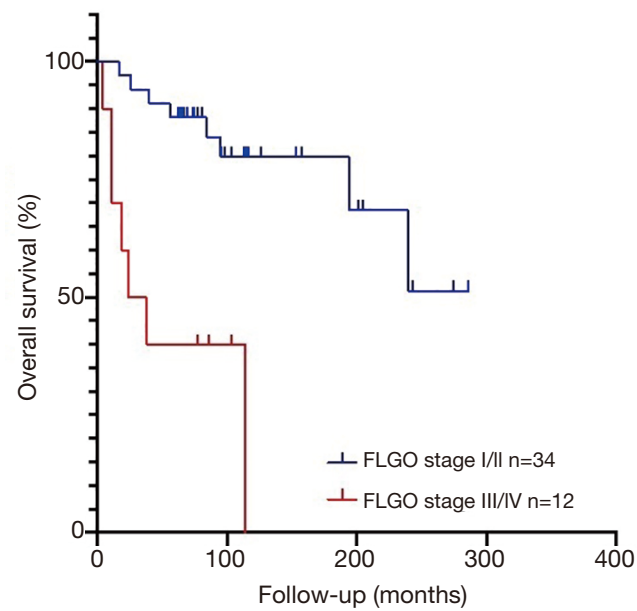

Data 3

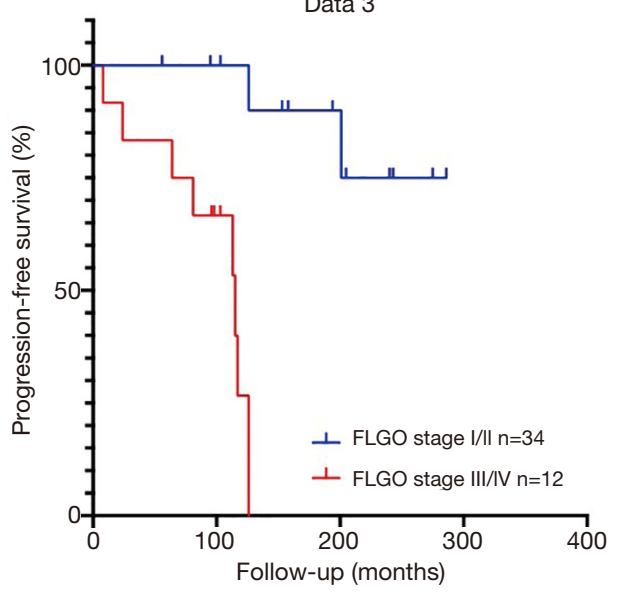

Data 2

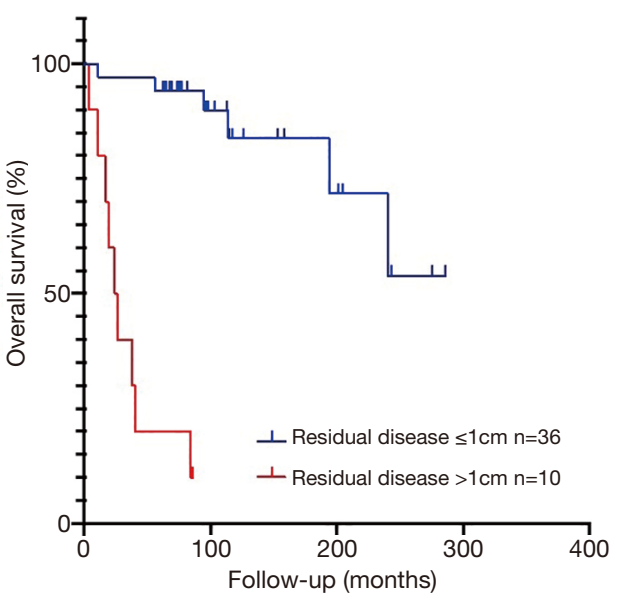

Data 4

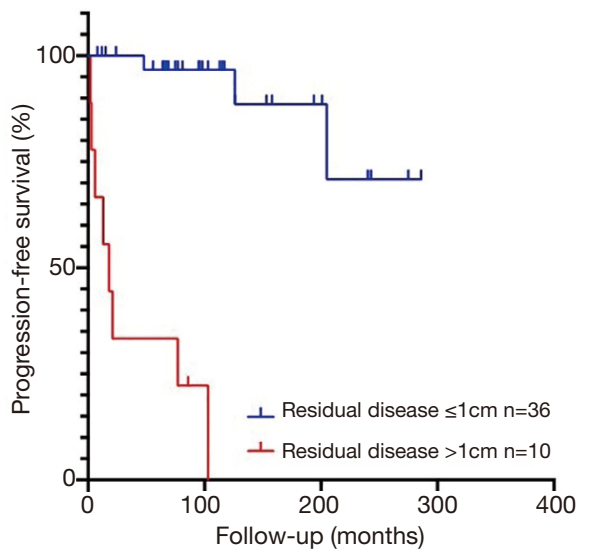

Figure 1 Survival curves of prognostic factors for OS and PFS. Data 1, OS by FIGO stage. Data 2, OS by residual disease. Data 3 , PFS by FIGO stage. Data 4, DFS residual disease. OS, overall survival; FIGO, International Congress of Gynecology and Obstetricians; PFS, progression-free survival.

and abnormal bloody-watery discharge.

The main treatment of PFTC is surgery. For the fallopian tube cancer confined in the pelvic cavity, a sample of the ascitic or peritoneal lavage fluid should be taken for cytological examination. The peritoneum should be explored comprehensively, and the peritoneum and adhesions that may have a metastasis should be biopsied or removed. Hysterectomy plus bilateral salpingooophorectomy lead to complete resection of the tumor as far as possible to avoid intraoperative tumor rupture, resection of the greater omentum, pelvic lymph nodes, and paraaortic lymph nodes (including lymph nodes on the surface of inferior vena cava and abdominal aorta).
For patients with tumors present beyond the scope of the pelvic cavity, CRS should be performed to make the diameter of the residual tumor less than $1 \mathrm{~cm}$. In order to achieve satisfactory CRS, partial bowel, bladder, spleen, liver, appendix, and peritoneum can be removed according to the individual clinical picture. For mucinous carcinoma, an appendectomy is necessary. For some patients who underwent tumor reduction surgery, the catheter was placed after the first operation for intraperitoneal chemotherapy.

In this study, all 46 patients were treated with surgery. There were 19 patients treated with comprehensive staging surgery, 25 patients were treated with satisfactory CRS, and two patients underwent salpingectomy (for 
choriocarcinoma). Postoperative residual tumor size showed no gross residual tumor in 32 cases, residual tumor diameter $\leq 1 \mathrm{~cm}$ in four cases, and residual tumor diameter $>1 \mathrm{~cm}$ in 10 cases.

Chemotherapy is the most important adjuvant therapy for PFTC and can also be used for the treatment of tumor recurrence (23). Platinum combined with paclitaxel has been used as the main chemotherapy regimen. The commonly used chemotherapy regimens include TP/TC/PC (cisplatin + cyclophosphamide). In this study, 40 patients received postoperative IV chemotherapy, with TP or TC regimen used after 2003 and PAC regimen used before 2003. Immature teratoma was treated with the bleomycin+VP16+cyclophosphamide regimen. Choriocarcinoma was used with 5-FU+KSM. Neuroendocrine carcinoma was treated with VP-16+cisplatin. 22 patients undertook intraperitoneal chemotherapy. The liver and kidney function, and medullary hematopoiesis function, were evaluated before and after chemotherapy. The patients without contraindication to chemotherapy were continued with the next cycle of chemotherapy. The levels of CA125 and other tumor markers were monitored during chemotherapy. The size of the residual tumor was monitored by pelvic ultrasound to evaluate the therapeutic effect.

There is much debate over the best timing for surgery and the best approach to delivering the chemotherapy (23). Although these debates continue, novel targeted therapies, including bevacizumab and poly [adenosine diphosphate (ADP)-ribose] polymerase (PARP) inhibitors, have emerged. PARP inhibitors are particularly efficacious in patients with $B R C A 1 / 2$ gene mutations, and their use has been shown to prolong patient survival (23).

In this study, 13 clinical variables (including age, postmenopausal period year, preoperative serum CA125 level, histologic subtype, FIGO stage, ascites tumor cells, surgical method, residual disease, lymphadenectomy, omentectomy, appendectomy, and chemotherapy course) were selected for Cox univariate analysis. Variables that had a significant difference in univariate analysis were applied in multivariate analysis.

The literature reports that the FIGO stage is a related factor of prognosis (3). In our study, FIGO stages were an independent prognostic factor both for OS $(\mathrm{P}=0.007)$ and PFS $(\mathrm{P}=0.003)$. This was similar to most studies in the literature (6). The later the pathological surgical stage is, the worse the prognosis is. However, there was no statistical difference in FIGO staging in multivariate analysis, indicating that FIGO surgical pathological staging interacts with other factors. Although it impacts the prognosis, it is not an independent prognostic factor in this study model. In Cox multivariate analysis, only satisfactory CRS could reduce the risk of recurrence and death, while FIGO staging had no significant difference.

The size of the residual tumor is a key factor affecting the prognosis (26). At present, it is considered that satisfactory CRS (residual tumor diameter less than $1 \mathrm{~cm}$ ) can improve the survival of patients, including OS, and PFS. A large number of retrospective clinical analyses have reached similar conclusions (26-28). In our series, Cox univariate and multivariate analysis showed that residual tumor size significantly affected OS and PFS (univariate analysis $\mathrm{P}=0.000$, multivariate analysis $\mathrm{P}=0.002$ ).

In our study, 25 patients underwent pelvic lymphadenectomy. The five-year OS and five-year PFS for the pelvic lymphadenectomy group showed no significant difference to that of the group without pelvic lymphadenectomy. These results are similar to the results of Klein et al. (29). Most studies failed to identify a correlation between prognosis and omentectomy $(15,30,31)$. Ramirez et al. suggested that appendectomy should not be routinely recommended in a study on early-stage ovarian cancer as it was not associated with survival (32). In our study, the univariate and multivariate analysis revealed no significant difference between these factors concerning survival in patients with PFTC.

FIGO guidelines on the overall staging surgery for primary ovarian cancer, fallopian tube cancer, and peritoneal cancer recommended ascites and peritoneal lavage fluid cytology as one of the bases of surgical pathological staging. However, there are different views on the significance of positive cytology of tumor cells in ascitic or peritoneal lavage fluid. Bakkum-Gamez et al. conducted a multivariate analysis of 161 patients with FIGO-staged EOC and found that capsule rupture $(\mathrm{P}=0.001)$ and positive tumor cytology of flushing fluid $(\mathrm{P}<0.001)$ were independent prognostic factors for PFS (33).

CRS followed by chemotherapy for the residual tumor has been recommended as a routine treatment of PFTC for decades (34). Several recent studies suggested that the platinum and paclitaxel-based chemotherapy regimen (TP) should be taken as the standard treatment in patients with PFTC (35). Our patients were followed up for a long time, as long as 20 years. The chemotherapy regimens before and after 2003 were different. Before 2003, intraperitoneal 
chemotherapy and IV chemotherapy were often used. After 2003, IV chemotherapy was mainly used, and a TP regimen was adopted.

\section{Conclusions}

This retrospective study followed up patients long-term for up to 20 years. There were, however, inconsistencies in the initial operation, staging, chemotherapy, and treatment time, which led to skewed results. The study was also limited by its sample size. We suggest that any postmenopausal women with vaginal bleeding, vaginal discharge, or lower abdominal pain should be alert to PFTC and undergo tumor markers and imaging examination as soon as possible to improve the early diagnosis rate of the disease. The mainstay of treatment is surgical cytoreduction and platinum-based chemotherapy. Whether the operation method is a comprehensive staging operation or CRS, achieving satisfactory R0 can improve OS and PFS. It is important to sample ascitic fluid to identify tumor cells to help forecast PFS. In the future, using these measures along with the anti-VEGF antibody, PARP inhibitors, and immunotherapy can help patients live longer, healthier lives.

\section{Acknowledgments}

Funding: This study was endorsed by the Shanghai Municipal Commission of Health and Family Planning (No.2017ZZ02016).

\section{Footnote}

Reporting Checklist: The authors have completed the STROBE reporting checklist. Available at https://dx.doi. org/10.21037/apm-21-2083

Data Sharing Statement: Available at https://dx.doi. org/10.21037/apm-21-2083

Conflicts of Interest: All authors have completed the ICMJE uniform disclosure form (available at https://dx.doi. org/10.21037/apm-21-2083). All authors report funding support from the Shanghai Municipal Commission of Health and Family Planning (No.2017ZZ02016). The authors have no other conflicts of interest to declare.

Ethical Statement: The authors are accountable for all aspects of the work in ensuring that questions related to the accuracy or integrity of any part of the work are appropriately investigated and resolved. All procedures performed in this study involving human participants were in accordance with the Declaration of Helsinki (as revised in 2013). The study was approved by ethic committee of Ren Ji Hospital, School of Medicine, Shanghai Jiao Tong University (NO.20200704) and informed consent was taken from all the patients.

Open Access Statement: This is an Open Access article distributed in accordance with the Creative Commons Attribution-NonCommercial-NoDerivs 4.0 International License (CC BY-NC-ND 4.0), which permits the noncommercial replication and distribution of the article with the strict proviso that no changes or edits are made and the original work is properly cited (including links to both the formal publication through the relevant DOI and the license). See: https://creativecommons.org/licenses/by-nc-nd/4.0/.

\section{References}

1. Kalampokas E, Sofoudis C, Boutas I, et al. Primary fallopian tube carcinoma: a case report and mini-review of the literature. Eur J Gynaecol Oncol 2014;35:595-6.

2. Pectasides D, Pectasides E, Economopoulos T, et al. Fallopian tube carcinoma: a review. Oncologist 2006;11:902-12.

3. Riska A, Leminen A. Updating on primary fallopian tube carcinoma. Acta Obstet Gynecol Scand 2007;86:1419-26.

4. Riska A, Leminen A. Determinants of incidence of primary fallopian tube carcinoma (PFTC). Methods Mol Biol 2009;472:387-96.

5. Rosenblatt KA, Weiss NS, Schwartz SM, et al. Incidence of malignant fallopian tube tumors. Gynecol Oncol 1989;35:236-9.

6. Stewart SL, Wike JM, Foster SL, et al. The incidence of primary fallopian tube cancer in the United States. Gynecol Oncol 2007;107:392-7.

7. Clayton NL, Jaaback KS, Hirschowitz L, et al. Primary fallopian tube carcinoma - the experience of a UK cancer centre and a review of the literature. J Obstet Gynaecol 2005;25:694-702.

8. Riska A, Leminen A, Pukkala E, et al. Sociodemographic determinants of incidence of primary fallopian tube carcinoma, Finland 1953-97. Int J Cancer 2003;104:643-5.

9. Pfeiffer P, Mogensen H, Amtrup F, et al. Primary carcinoma of the fallopian tube. A retrospective study of 
patients reported to the Danish Cancer Registry in a fiveyear period. Acta Oncol 1989;28:7-11.

10. Hellström AC, Silfverswärd C, Nilsson B, et al. Carcinoma of the fallopian tube. A clinical and histopathologic review. The Radiumhemmet series. Int J Gynecol Cancer 1994;4:395-400.

11. Trabert B, Coburn SB, Mariani A, et al. Reported Incidence and Survival of Fallopian Tube Carcinomas: A Population-Based Analysis From the North American Association of Central Cancer Registries. J Natl Cancer Inst 2018;110:750-7.

12. Pereira A, Pérez-Medina T, Magrina JF, et al. International Federation of gynecology and obstetrics staging classification for cancer of the ovary, fallopian tube, and peritoneum: estimation of survival in patients with nodepositive epithelial ovarian cancer. Int J Gynecol Cancer 2015;25:49-54.

13. Lee KR, Tavassoli FA, Prat J, et al. Surface epithelialstromal tumors. In: Tavassoli FA, Devilee P. Editors. Pathology \& Genetics of Tumors of the Breast and Female Genital Organs, IARC Press, Lyon. 2003.

14. Shimizu Y, Kamoi S, Amada S, et al. Toward the development of a universal grading system for ovarian epithelial carcinoma: testing of a proposed system in a series of 461 patients with uniform treatment and followup. Cancer 1998;82:893-901.

15. Alvarado-Cabrero I, Stolnicu S, Kiyokawa T, et al. Carcinoma of the fallopian tube: Results of a multiinstitutional retrospective analysis of 127 patients with evaluation of staging and prognostic factors. Ann Diagn Pathol 2013;17:159-64.

16. Rosen A, Klein M, Lahousen M, et al. Primary carcinoma of the fallopian tube--a retrospective analysis of 115 patients. Austrian Cooperative Study Group for Fallopian Tube Carcinoma. Br J Cancer 1993;68:605-9.

17. Bao L, Ding Y, Cai Q, et al. Primary Fallopian Tube Carcinoma: A Single-Institution Experience of 101 Cases: A Retrospective Study. Int J Gynecol Cancer 2016;26:424-30.

18. Sonmezer M, Ustun Y, Gungor M, et al. Primary carcinoma of the fallopian tube in a 88 years old woman: review of the literature. Indian J Cancer 2001;38:61-4.

19. Liu L, Xu X, Jia L, et al. Primary fallopian tube carcinoma-a retrospective analysis of 66 cases. Eur J Gynaecol Oncol 2015;36:161-7.

20. Kindelberger DW, Lee Y, Miron A, et al. Intraepithelial carcinoma of the fimbria and pelvic serous carcinoma:
Evidence for a causal relationship. Am J Surg Pathol 2007;31:161-9.

21. Labidi-Galy SI, Papp E, Hallberg D, et al. High grade serous ovarian carcinomas originate in the fallopian tube. Nat Commun 2017;8:1093.

22. Kim J, Park EY, Kim O, et al. Cell Origins of High-Grade Serous Ovarian Cancer. Cancers (Basel) 2018. [Epub ahead of print] doi:10.3390/cancers10110433.

23. Stasenko M, Fillipova O, Tew WP, et al. Fallopian Tube Carcinoma. J Oncol Pract 2019;15:375-82.

24. Pettersson F. Staging rules for gestational trophoblastic tumors and fallopian tube cancer. Acta Obstet Gynecol Scand 1992;71:224-5.

25. Wethington SL, Herzog TJ, Seshan VE, et al. Improved survival for fallopian tube cancer: a comparison of clinical characteristics and outcome for primary fallopian tube and ovarian cancer. Cancer 2008;113:3298-306.

26. Ul'rikh EA, Papunidi MD, Urmancheeva AF, et al. [Fallopian tube carcinoma: clinical and morphological features, analysis of 69 cases]. Vopr Onkol 2014;60:375-8.

27. Baekelandt M, Jorunn Nesbakken A, Kristensen GB, et al. Carcinoma of the fallopian tube. Cancer 2000;89:2076-84.

28. Rosen AC, Klein M, Hafner E, et al. Management and prognosis of primary fallopian tube carcinoma. Austrian Cooperative Study Group for Fallopian Tube Carcinoma. Gynecol Obstet Invest 1999;47:45-51.

29. Klein M, Rosen A, Lahousen M, et al. Radical lymphadenectomy in the primary carcinoma of the fallopian tube. Arch Gynecol Obstet 1993;253:21-5.

30. Shamshirsaz AA, Buekers T, Degeest K, et al. A singleinstitution evaluation of factors important in fallopian tube carcinoma recurrence and survival. Int J Gynecol Cancer 2011;21:1232-40.

31. Riska A, Alfthan H, Finne P, et al. Preoperative serum hCGbeta as a prognostic marker in primary fallopian tube carcinoma. Tumour Biol 2006;27:43-9.

32. Ramirez PT, Slomovitz BM, McQuinn L, et al. Role of appendectomy at the time of primary surgery in patients with early-stage ovarian cancer. Gynecol Oncol 2006;103:888-90.

33. Bakkum-Gamez JN, Richardson DL, Seamon LG, et al. Influence of intraoperative capsule rupture on outcomes in stage I epithelial ovarian cancer. Obstet Gynecol 2009;113:11-7.

34. Mikkelsen MS, Christiansen T, Petersen LK, et al. Morbidity after cytoreductive surgery and hyperthermic intraperitoneal chemotherapy with carboplatin used for 
ovarian, tubal, and primary peritoneal cancer. J Surg Oncol 2019;120:550-7.

35. Gemignani ML, Hensley ML, Cohen R, et al. Paclitaxelbased chemotherapy in carcinoma of the fallopian tube.

Cite this article as: $\mathrm{Li} \mathrm{S,} \mathrm{Yu} \mathrm{M,} \mathrm{Bai} \mathrm{W,} \mathrm{Shi} \mathrm{J,} \mathrm{Di} \mathrm{W.} \mathrm{Long-}$ term follow-up of 46 cases of primary fallopian tube carcinoma: a single institute study. Ann Palliat Med 2021;10(8):9122-9135. doi: 10.21037/apm-21-2083
Gynecol Oncol 2001;80:16-20.

(English Language Editors: M. Bucci and J. Chapnick) 